Article

\title{
Biochemical Change at the Setting-up of the Crossed-Lamellar Layer in Nerita undata Shell (Mollusca, Gastropoda)
}

\author{
Julius Nouet $^{1, \star}$, Marine Cotte ${ }^{2}$, Jean-Pierre Cuif ${ }^{1}$, Yannicke Dauphin ${ }^{1}$ and Murielle Salomé ${ }^{2}$ \\ ${ }^{1}$ IDES - UMR8148, University Paris-Sud, Orsay 91405, France; \\ E-Mails: jean-pierre.cuif@u-psud.fr (J.P.C.); yannicke.dauphin@u-psud.fr (Y.D.) \\ ${ }^{2}$ European Synchrotron Radiation Facility, BP220, Grenoble Cedex F-38043, France; \\ E-Mails: marine.cotte@esrf.fr (M.C.); salome@esrf.fr (M.S.) \\ * Author to whom correspondence should be addressed; E-Mail: julius.nouet@u-psud.fr; \\ Tel.: +33-01-69-15-61-21; Fax: +33-01-69-15-61-23.
}

Received: 28 January 2012; in revised form: 24 February 2012 / Accepted: 21 March 2012 /

Published: 29 March 2012

\begin{abstract}
Nerita undata is a marine gastropod, the shell of which consists of an external layer composed of very fine, long and undulating calcite prisms, and of an internal aragonite crossed-lamellar layer. As for any Ca-carbonate shell, both layers are composite materials, resulting from the sub-micrometric association of organic macromolecules with the mineral phase. But at the transition between the two layers, in situ synchrotron-based mapping using $\mu$-XANES spectroscopy performed at the S K-edge and SR-FTIR spectroscopy reveals that biochemical compositions change correlatively with the mineral phase, such as displayed by the distribution of sulfur-containing organic compounds (S-polysaccharides or S-amino acids) and organic molecular groups (amide I and II bands). These results highlight the complex change of secretory activity operated by the mineralizing tissue (the mollusk mantle) between these two parts of the shell, which is suspected to minutely control the setting-up of the crossed-lamellar microstructural pattern over the calcite prisms-A not so straightforward feature.
\end{abstract}

Keywords: biomineralization; crossed-lamellar; XANES; SR-FTIR 


\section{Introduction}

Molluscan shells display a wide variety of acellular structures and morphologies. Mineralized by a dedicated area of the mollusc mantle, they are layered and composed of one or several Ca-carbonate polymorph(s) (usually calcite or aragonite; vaterite is occasionally found), which can present a wide variety of morphologies and structural organizations. More than 10 microstructural types have been described [1-3], their respective size, shape and arrangements within the shell being highly taxonomically dependent.

But these crystallized exoskeletons are also long known to be composite materials: they indeed incorporate many organic macromolecules, that are associated to the mineral phase down to a fine scale [4-9]. These organic compounds are secreted by a mineralizing tissue, a dedicated part of the mollusc mantle epithelium. They allow the mantle to exert a precise control over the mineralization of the shell, including mineral polymorph selection and structural organizations [10-12], by way of complex organo-mineral interactions occurring at very fine scale-which are yet to be deciphered. As a result, the different layers of mollusc's shells always present specific organic compositions, which are taxa-dependent but also closely related to the Ca-carbonate polymorph/microstructure expressed. Detailed observations of the transition between two shell layers, including minute description of the changes occurring both in microstructural patterns and biochemical compositions, are therefore very interesting to highlight the change of metabolic activity of the mollusc mantle during this shift of mineralization modalities.

This paper is focused at the shift between inner and outer layers of the shell of Nerita undata shell (Gastropoda, Neritopsina). This taxon is indeed quite representative of the diversity of structures commonly found within molluscan shells, as it is described as composed of an inner aragonite regular crossed-lamellar layer and an outer calcite prismatic layer [1]. Regular crossed-lamellar organization is, among the variety of microstructural types described, one of the most commonly found, often forming one or several shell layer(s) of many bivalves or gastropods. Its fundamental architecture consists of a nicely ordered arrangement of lamellae, perpendicular to the shell growth surface and around 5 to $30 \mu \mathrm{m}$ thick (usually called 1st order lamellae), that are themselves composed of sheet-like arrangements (2nd order lamellae) of submicrometric, elongated individual rods or fibers (3rd order lamellae). The 2nd order lamellae are dipping in opposite directions between two consecutive first order lamellae (typical angle comprised between $90^{\circ}$ and $130^{\circ}$, [13]). The calcite layer is usually described as very colored and composed of fine, irregular prisms, undulating in a peculiar manner [1].

Various techniques, including X-ray Absorption Near Edge Structure (XANES) and Fourier-transform Infra-red (FT-IR) spectromicroscopies, are used to characterize the distribution of the organic components in correlation with the microstructural changes, which follow the setting-up of crossed-lamellar pattern over the calcite prisms. Comparison with previous study [14] of the setting-up of aragonite nacreous tablets over calcite prisms within the shell of Pinctada margaritifera (Bivalvia, Pteriomorphia) would highlight specific biochemical compositions/distributions, but also some common features. 


\section{Results and Discussion}

\subsection{Results}

\subsubsection{General Microstructural Organization}

Polarized and analyzed Light Microscopy (PLM) and Scanning Electron Microscopy (SEM) were used for general orientation and shell microstructure characterization (Figure 1). As shown in Figure 1a,b crossed-lamellar layer is found to be precisely ordered, with straight and long 1st order lamellae that present the alternate orientation, so typical of this microstructural organization (Figure 1b). The shift from calcite outer layer to aragonite inner layer appears to be fine and sharp at low magnification, and straight along the shell section (Figure 1b). The calcite prisms, however, are found to be highly irregular, grouped in wavy fagots with distinct orientations. This layer is said to be "very irregularly prismatic; the prisms, ... are mostly very fine and undulating in a peculiar manner" [1, p. 303]. SEM observations of the preparation presenting both radial and transverse sections (Figure 1c,d), cut close to the beginning of crossed-lamellar deposition, highlight the regular 3D arrangement of 1st order lamellae and the alternate orientation of the 2nd order lamellae, with an angle fitting with other taxa [13].

Figure 1. Microstructural organization of a Nerita shell. (a) PLM view of an ultra-thin radial section of the outer lip of the peristome of the shell, showing the outer calcite prisms layer (bottom) and inner aragonite crossed-lamellar (top). Black square marks the localization of Figure 1b. Blue square corresponds to the approximate location of the radial cut, which is colored in blue in Figure 1c; (b) PLM view of the shift from the calcite prisms layer (C) to the aragonite crossed-lamellar layer (A), which presents a conserved crystal orientation one lamella over two; (c) SEM image of a fragment of the peristome, cut along both radial and transverse sections, each side polished and etched to reveal calcite (C) and aragonite (A) microstructural patterns. White square marks the localization of Figure 1d; (d) SEM image of same fragment at the shift from the outer calcite layer $(\mathrm{C})$ to the inner aragonite layer (A). White square marks the localization of Figure 1e. Green arrows mark a growth layer; (e) SEM image of the aragonite crossed-lamellar inner layer (A) of the same fragment. Green arrows mark a growth layer. Stars mark individual 1st order lamellae; (f) and (g) SEM (BSE detector) images of a radial polished section at the shift from calcite (C) to aragonite (A) layer, stained with $\mathrm{Pb}$ citrate and uranyl acetate. Green arrows mark a growth layer. White square marks the localization of Figure 1h; (h) SEM (BSE detector) images of a radial polished section of aragonite crossed-lamellar layer. Stars mark individual 1st order lamellae. Arrows mark a growth layer; (i,j) SEM image of a broken, unetched fragment showing (i) the irregular, fiber-like shape of the calcite prisms forming the outer layer, (j) the sheet-like arrangement (2nd order lamellae) of 3rd order rods that form two adjacent 1 st order lamellae (white stars). 

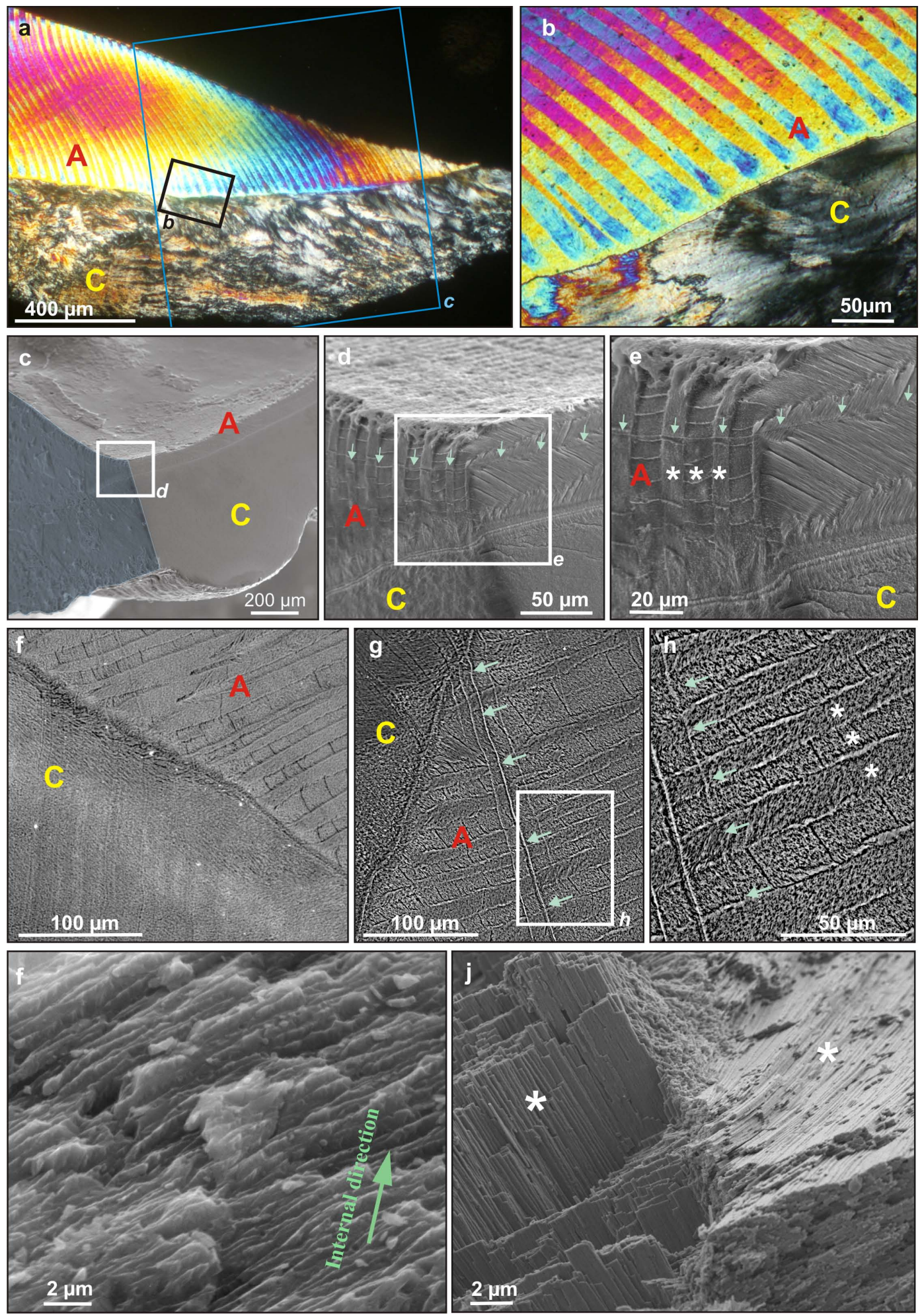

SEM heavy metals staining procedure used for observations with Back Scattered Electrons (BSE) detector is found to enhance organic/mineral contrast, and therefore microstructural patterns (Figure $1 \mathrm{f}-\mathrm{h}$ ). Growth lines, perpendicular to 1 st order rods, are thus clearly marked (Figure 1g,h) when they could only be faintly distinguished in Figure 1e. Some areas locally enriched in organic compounds (white contrast) can also be evidenced, forming a fine layer at the shift from calcite to aragonite (Figure 1g), or localized between each 1st order lamellae in crossed-lamellar (Figure 1h). 
At higher magnification, the traditional sheet-like arrangement (2nd order lamellae) of 3rd order rods, the orientation of which shifts between each 1st order lamella, can easily be identified (Figure 1j). The inner structure of the "prismatic" calcite layer is very different from the calcite prisms of Pinna and Atrina that are often used as a reference for this kind of structure: it is indeed composed of fiber-like, $\sim 1 \mu \mathrm{m}$ thick and undulating units (Figure 1i). For consistency with the literature, they will still be called "prism" from now on.

\subsubsection{XANES—Sulfur Speciations and Elemental Compositions Distributions}

$\mu$-XANES can be used to map the distribution of sulfur species and some elements content at high spatial resolution [14-18].

Herein, standards compounds rich in sulfur were scanned around S-K absorption edge between 2,450 and 2,540 eV, in unfocused mode, to provide reference spectra for later maps. Chondroitin sulfate was used as a standard for S-containing polysaccharides and gypsum as a standard for inorganic sulfates; methionine, cysteine and cystine were used as standards for S-containing amino acids. The S K-edge spectra of methionine, cysteine and cystine show a main peak at 2,473 eV (Figure 2a). Both spectra of chondroitin sulfate and gypsum (Figure $2 \mathrm{~b}$ ) present a main absorption peak at 2,482 eV, but display specific EXAFS profiles. Spectra from inner and outer layers of the shell (Figure 2c) both display a profile, which is matching EXAFS spectrum for chondroitin sulfate standard, but not for inorganic sulfate standard: this reveals that the $\mathrm{S}$ present in both layers of the shell is associated to organic molecules, mostly organic sulfates. Energies at 2,482 and 2,473 eV were therefore selected to map the repartition of S-containing organic compounds within the following 2D scans.

The map for S-polysaccharides at low magnification (energy $=2,482 \mathrm{eV}$ ) presents much higher intensities in the calcite layer (bottom) than in the aragonite layer (top) (Figure 2d). Fluorescence maps acquired simultaneously show that the calcite layer is also richer in $\mathrm{Sr}$ and $\mathrm{P}$ contents (Figure 2e,f). Maps for the distribution of $\mathrm{S}$ containing amino acids (energy $=2,473 \mathrm{eV}$ ) also present lowered intensities within the aragonite layer (Figure $2 \mathrm{~g}$ ) compared to calcite layer (Figure 2k).

Within the calcite layer, the distributions of S-polysaccharides (Figure 2d,i), Sr (Figure 2e,m), $\mathrm{P}$ (Figure 2f,n) and S-amino acids (Figure 2k) present marked bands, corresponding to the growth layering of the shell. Within the aragonite layer, growth layering is more faintly marked but can still be distinguished on S-polysaccharides maps (Figure 2d,h) and $\mathrm{Sr}$ (Figure 2e,i), whereas it remains unseen on $\mathrm{P}$ (Figure 2f,j) and S-amino acids maps (Figure $2 \mathrm{~g}$ ). The aragonite layer also presents an heterogeneous distribution of S-polysaccharides following the crossed-lamellar microstructure, the $1^{s t}$ order lamellae being richer at heart than on their respective boundaries (Figure $2 \mathrm{~h}$ ). 
Figure 2. Sulfur speciations and elemental maps. (a) and (b) XANES spectra of S reference compounds. (a) Methionine, cysteine and cystine amino acids spectra all display a main peak at 2,473 eV; (b) Chondroitin sulfate and $\mathrm{CaSO}_{4}$ display a main peak at 2,482 eV, with specific EXAFS patterns; (c) Spectra extracted from calcite and aragonite layers, matching chondroitin sulfate EXAFS pattern; $(\mathbf{d}-\mathbf{f})$ Maps of the transition between inner calcite (bottom) and outer aragonite (top) layers; (d) S-polysaccharides map, performed at 2,482 eV, showing a higher intensity within the calcite layer, as well as marked growth layering. Red and green circles mark the locations, from which Figure 2c spectra were extracted; (e) Sr map performed at 2,482 eV; (f) P map performed at 2,482 eV; (g-j) Maps of the aragonite crossed-lamellar layer; (g) Amino acids-linked S map, performed at 2,473 eV; (h) S-polysaccharides map, performed at 2,482 eV; (i) Sr map performed at 2,482 eV; (j) P map performed at 2,482 eV; $(\mathbf{k}-\mathbf{n})$ Maps of the calcite layer, all displaying marked growth layering and higher intensities than maps $2 \mathrm{~g}-\mathrm{j}$; (k) Amino acids-linked $\mathrm{S}$ map, performed at 2,473 eV; (I) S-polysaccharides map, performed at 2,482 eV; (m) Sr map performed at 2,482 eV; (n) P map performed at 2,482 eV.
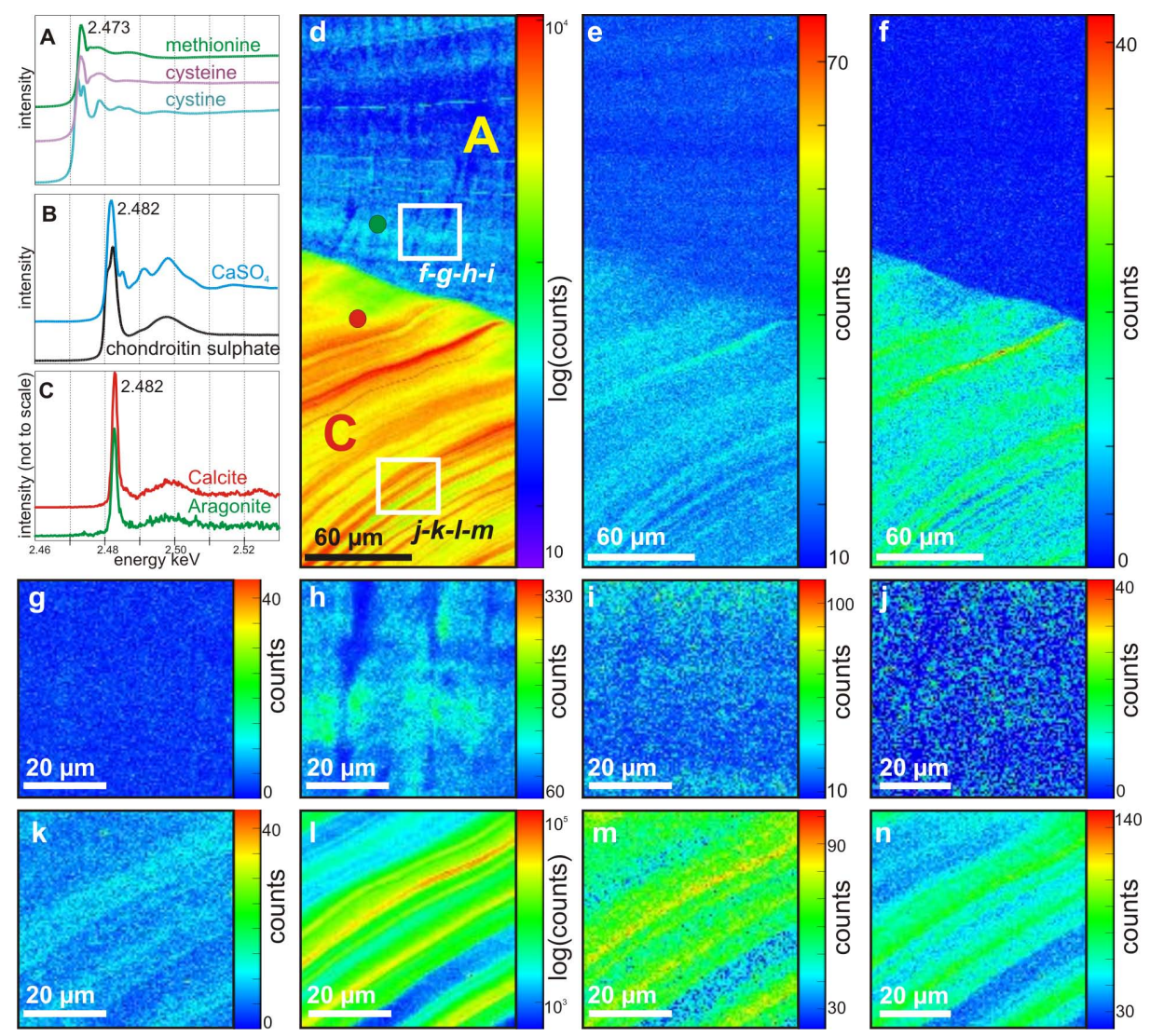

\subsubsection{FT-IR Maps—Distribution of Organic and Mineral Bands}

Calcite and aragonite FT-IR spectra present characteristic bands, attributed to $\mathrm{CO}_{3}^{2-}$ group vibrations: $\nu_{3}$ band at $\sim 1,428 \mathrm{~cm}^{-1}$, the $\nu_{2}$ doublet at $877-848 \mathrm{~cm}^{-1}, \nu_{4}$ band at $713 \mathrm{~cm}^{-1}$ are specific for calcite, whereas $\nu_{3}$ band at $\sim 1,471 \mathrm{~cm}^{-1}$ and the two doublets, $\nu_{2}$ at $858-844 \mathrm{~cm}^{-1}$ and $\nu_{4}$ at $713-700 \mathrm{~cm}^{-1}$, are specific for aragonite [19]. 
Figure 3. FT-IR maps. (a) Localization of the 2D scan on a polished radial section, at the transition between the outer calcite prisms layer (bottom) and the inner aragonite crossed-lamellar (top); (b) IR map of the $\nu_{2}$ band of aragonite at $864 \mathrm{~cm}^{-1}$; (c) IR map of the $\nu_{3}$ band of calcite at $1,428 \mathrm{~cm}^{-1}$; (d) IR map of the amide II band at $1,542 \mathrm{~cm}^{-1}$, which is enriched in calcite layer; (e) IR map of the amide II band at $1,573 \mathrm{~cm}^{-1}$, which is enriched in aragonite layer, one 1st order lamella on two; (f and $\mathbf{g}$ ) IR maps of amide I bands at (f) $1,599 \mathrm{~cm}^{-1}$ and (g) $1,636 \mathrm{~cm}^{-1}$, both enriched in the same 1 st order lamellae (each one of two), but anti-correlated to the distribution of map $3 \mathrm{e}$.
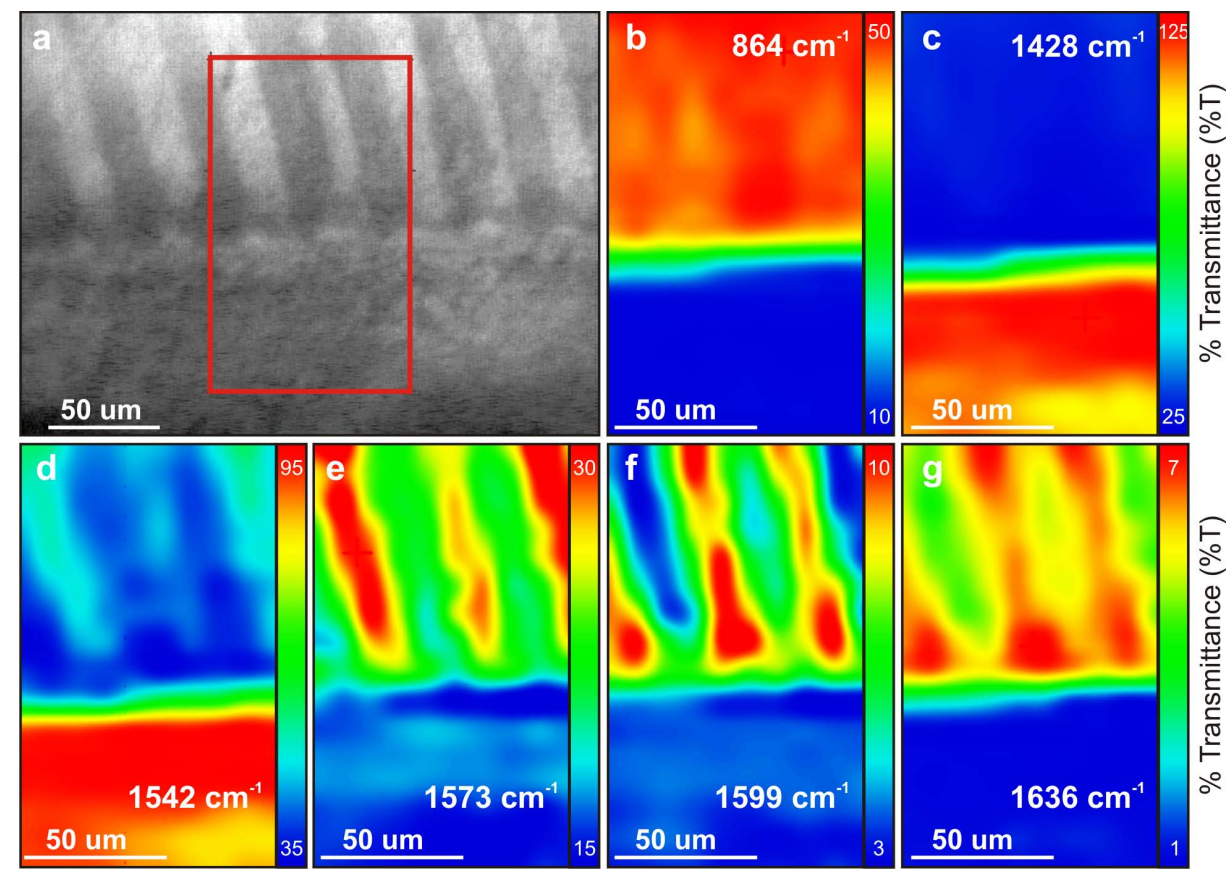

FT-IR scan was performed at the limit between inner and outer layer (Figure 3a). A band is found at $864 \mathrm{~cm}^{-1}$ within the inner layer (Figure $3 \mathrm{~b}$ ), which is compatible with the mean position of the $\nu_{2}$ band in molluscan aragonite presenting low Sr content [20,21]. Map at 1,428 $\mathrm{cm}^{-1}$ confirms that the outer layer is calcitic (Figure 3c). To note, the lowered intensities recorded at the shift from calcite to aragonite reflect a mixed signal (most probably caused by the tilt of this boundary, combined with the unknown depth of IR response).

A response for $\mathrm{N}$-containing organic compounds is also recorded within the range for amide I $\left(1,597-1,695 \mathrm{~cm}^{-1}\right)$ and amide II $\left(1,515-1,580 \mathrm{~cm}^{-1}\right)$ bands. In particular, map at $1,542 \mathrm{~cm}^{-1}$ shows that calcite layer is richer than aragonite layer in amide II bands (Figure 3d), whereas maps at 1,599 and $1,636 \mathrm{~cm}^{-1}$ (Figure 3f,g) show that amide I bands are richer in aragonite layer: those results confirm a shift of organic composition, simultaneously to the calcite/aragonite transition.

Most interesting feature is observed within aragonite layer, where an alternate distribution of several organic bands (at 1,573, 1,599 and 1,636 $\mathrm{cm}^{-1}$ ) exactly match inter-crossed orientation of 1st order lamellae (Figure $3 \mathrm{e}-\mathrm{g}$ ). Band at $1,573 \mathrm{~cm}^{-1}$ is within amide II range, and might be assigned to aspartic acid $(\mathrm{COOH})$ [22]; amide I vibrations between 1,640 and $1,620 \mathrm{~cm}^{-1}$ are usually ascribed to $\beta$-sheet conformation $[23,24]$. This alternate distribution of organic bands is seen one every two lamellae for those three wavenumbers, but is anti-correlated between amide I bands and the band at $1,573 \mathrm{~cm}^{-1}$ : the lamellae, the richer at $1,573 \mathrm{~cm}^{-1}$ are also the poorer at 1,599 and $1,636 \mathrm{~cm}^{-1}$, and vice versa. 


\subsubsection{Setting-up of Crossed-Lamellar Pattern}

A closer look was given to the boundary between outer and inner layers, revealing a rather complex mechanism before the setting-up of a regular crossed-lamellar pattern. The calcite prisms are delimited by a seemingly continuous layer, which displays a strong autofluorescence signal under laser confocal microscope (Figure 4c,d). The latter indicates that this layer is highly enriched in autofluorescent organic compounds, which probably form an organic mesh or membrane. As seen in thin section in PLM (Figure 4b), this sheet appears rather slim.

Figure 4. Setting-up of crossed-lamellar pattern. (a) PLM view of a thick radial section of the outer lip of the shell. A brownish, always extinct contrast, corresponding to areas enriched in organics, marks the growth layering, the limits between 1st order lamellae and a punctuated line at the transition between the calcite (C) and aragonite (A) layer; (b) Higher magnification PLM view of a thin section (5-10 $\mu$ m thick) of the transition between calcite prisms or fibers (C) and crossed-lamellar (A). Colors of polarization highlight the alternate orientation of 1 st order lamellae, and $2 \mathrm{nd} / 3 \mathrm{rd}$ order patterns can be distinguished. A very thin membrane can be seen at the transition from calcite to aragonite, followed by a layer of homogeneous greenish polarization color. Arrows mark growth layers; (c and d) Confocal laser microscopy image of the transition between the calcite (C) and aragonite (A) layers. A false color red LUT is used to highlight the high fluorescence contrast correlated to strong organic content; it reveals a continuous line, followed by a punctuated line at the transition. Green arrows mark a growth layer; (e-h) XRF maps of the intermediate area between inner calcite (bottom) and outer aragonite (top) layers; (e) S-polysaccharides map, performed at 2,482 eV; (f) Amino acids-linked S map, performed at 2,473 eV, showing a depleted content in the intermediate layer; (g) Sr map performed at 2,482 eV, showing a slightly enriched rim in the intermediate layer; (h) $\mathrm{P}$ map performed at 2,482 eV.

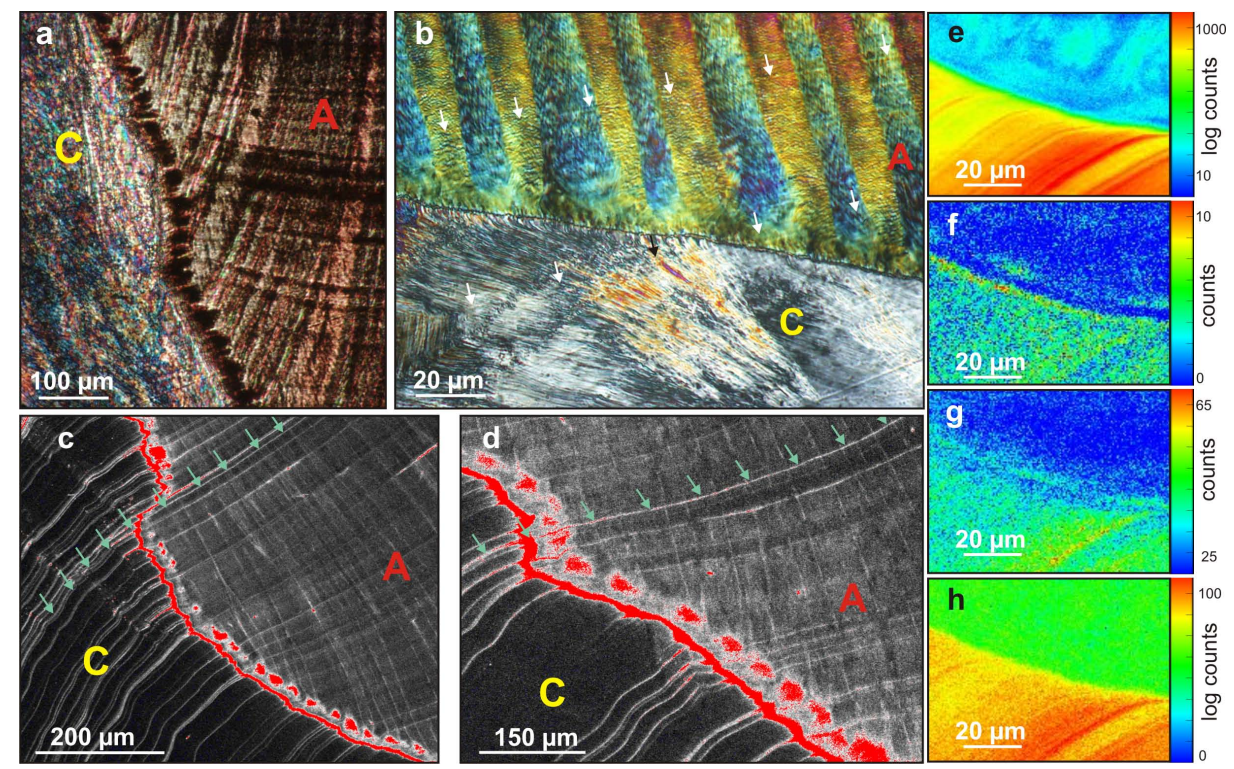

A dotted-pattern is also clearly visible under laser confocal microscope, and there seems to be a good correspondence between these "dots" richer in organics and the initiation of crossed-lamellar alternate orientation (Figure 4d). These dots are also visible, as well as growth layering, on the thick section 
under PLM (Figure 4a): they display a brownish, always extinct contrast, coherent with the presence of organic patches in these areas. This contrast is lost on the thinner section (Figure 4b), but on the other hand the morphology of the very beginning of 1st order lamellae is nicely highlighted, and displays an ovoid configuration every one lamella over two.

In between the thin membrane marking the shift from calcite to aragonite and the beginning of inter-crossed 1st order lamellae, an intermediate area less than $10 \mu \mathrm{m}$ thick is observed that does not display the sharp changes of crystallographic orientation so characteristic of crossed-lamellar layer in PLM (a continuous, greenish contrast is observed in Figure 4b). The 1st order lamellae displaying an alternate orientation, exactly conserved every two 1st order lamellae (blue contrast in Figure 4b), do only appear within the aragonite layer that is deposited just over this intermediate layer. The sheet-like arrangement (2nd order lamellae) of elongated 3rd order rods appears concurrently, and is afterward conserved through each growth increment of the shell, such as seen in Figure $4 \mathrm{~b}$.

Some punctuated structures might be discerned on XANES maps of the intermediate area for organic sulfates (Figure 4e), but are wanting for S-containing amino acids (Figure 4f); those are likely to correspond to the dotted pattern highlighted in Figure $4 \mathrm{c}, \mathrm{d}$ that would therefore be faintly enriched in S-polysaccharides. The organic mesh over the calcite is not specifically enriched in S-polysaccharides (a thin, homogeneous rim of intermediate value between calcite and aragonite may still be visible), and might be faintly enriched in S-containing amino acids, which is hard to determine due to low count at $2,473 \mathrm{eV}$ (Figure 4f). However, the intermediate layer on top of it is indubitably poorer in S containing amino acids (Figure 4f), it is slightly enriched in Sr compared to the aragonite crossed-lamellar layer (Figure $4 \mathrm{~g}$ ) and already displays the same intensity for $\mathrm{P}$ as aragonite crossed-lamellar (Figure 4h).

\subsection{Discussion and Conclusion}

\subsubsection{Shift in Biochemical Compositions}

Our results show that the shift between the two layers of $N$. undata shell is accompanied by a sharp change in organic compositions, such as already observed using similar techniques on molluscs with shells composed of several layers of distinct microstructural organizations and/or carbonate polymorph expressed [14,25]. But comparison with the shift from calcite prisms to aragonite nacreous layer in P. margaritifera shell (Bivalvia, Pteriomorphia) [25] shows that the analogy is not so straightforward: whereas the outer, calcite layers of both P. margaritifera and $N$. undata are enriched in S-polysaccharides compared to the inner, aragonite layer, $N$. undata's crossed-lamellar is also found to be poor in S-containing amino acids, whereas the nacreous layer of $P$. margaritifera is richer than its calcite prisms. No correlation can be established using amide bands either. P. margaritifera prisms display enriched bands in the amide I wavenumber range and nacreous inner layer is enriched in amide II bands [25]; but $N$. undata's outer layer is more enriched in amide II bands, and inner crossed-lamellar is enriched in amide I bands. This confirms the high specificity of organic compositions of mollusc shell layers that are strongly correlated to microstructural types. So far, the only feature that seems common to bivalve shells such as P. margaritifera or Pinna nobilis (Bivalvia, Pteriomorphia) [16], or gastropod shells such as Haliotis tuberculata (Gastropoda, Vetigastropoda) [14] or N. undata, is that the calcite "prisms" (as seen 
above, this appellation might not be well fitting for $N$. undata's outer layer) are always much enriched in S-polysaccharides compared to the other microstructural types present in other layers of the shells.

\subsubsection{Transition Sequence in Nerita undata}

Previous study on N. undata shell using Confocal Raman Microscopy (CRM) [26] already illustrated the specialization of the mantle epithelium, through the change in the secretion of polyenic pigments just before the shift from calcite to aragonite. Same study demonstrated that this shift is sharp $(<300 \mathrm{~nm})$, but immediately followed by an $\sim 10 \mu$ m thick aragonite layer, displaying a homogeneous crystallographic orientation. Our results confirm the presence of this intermediate area with no alternate crystallographic orientations, but a specific composition in S-containing amino acids and $\mathrm{Sr}$ content. However, new confocal and PLM results also reveal that this intermediate area is preceded by a very thin layer enriched in organic compounds, seemingly continuous under confocal microscope (thus probably forming a tight organic mesh, or membrane), the composition of which is still unknown, and that would exactly correspond to the sharp shift from calcite prisms or fibers to homogeneous aragonite.

The most striking feature is the presence of centers, enriched in organics, when the new alternate orientation of crossed-lamellar truly begins, one every two 1st order lamellae, as seen with PLM and confocal laser microscopy. This confirms previous results using CRM, where similar dots were observed, by mapping the distribution of $-\mathrm{CH}$ bands [26]. The $-\mathrm{CH}$ functional groups are found within many organic compounds, such as lipids for instance. Our new results show that these dots also contain some S-polysaccharides (Figure 4e), as well as N-containing molecules presenting amide I bands (Figure 3f,g).

Surprisingly, once the crossed-lamellar pattern is set up and is thereafter exactly conserved through each growth increment, the preferential secretion of compounds containing amide I bands, and possibly $\mathrm{COOH}$, is also observed one 1st order lamella on two (Figure 3f,g).

SEM observations also suggest that the boundaries between 1st order lamellae are faintly enriched is organics (Figure 1g,h); but according to XANES map at 2,482 eV, the latter would be deprived in S-polysaccharides (Figure 2h). Similar pattern was observed using CRM, enriched in compounds containing abundant $-\mathrm{CH}$ molecular groups [26]; but high resolution transmission electron microscopy (TEM) study did not evidence the presence of neither thick nor continuous membranes [27]. Instead, the presence of empty "holes" was observed, the latter being most probably remains of organic compounds burnt under the TEM. This suggests a punctuated limit, composed of patches of compounds rich in molecules containing - $\mathrm{CH}$ groups, and poor in S-polysaccharides and S-containing amino acids.

\subsubsection{Conclusions}

The succession of steps observed, which ultimately leads to the setting-up of the regular crossed-lamellar pattern of $N$. undata shell's inner layer, is quite similar to the setting-up of aragonite nacreous layer over the calcite prisms, which has been previously described within $P$. margaritifera's shell [25]. In both cases, the change of mineralogy is correlated to a change in organic compositions. Each time, it is preceded by a progressive change of biochemical compositions within the calcite layer [25,26], revealing that the mantle metabolism is already changing prior to the transition. The change of mineralogy itself is sharp, and followed by the deposition of a thin layer very enriched in 
organics that precedes any aragonite deposition. Then follows a distinct intermediate layer, a few tens of microns thick, that is already in aragonite but with specific trace elements/organics contents, and with microstructure features not found in any of the two layers. Only on top of this intermediate layer does the mechanism that builds the inner layer microstructural type really start (crossed-lamellar or nacre) and is thereafter cyclically continued through successive growth increments.

Of course, as the shells grow both in thickness and length, these steps occur in fact simultaneously, on the inner growing face of the shell. This reveals the minute specialization of the mineralizing epithelium of molluscs mantle. The latter is indeed composed of dedicated areas that mineralize each layer; each one by means of a specific metabolism, which produces its own assemblage of organic compounds. The whole growing edge of the shell, including both layers, is therefore produced synchronously, through the simultaneous secretion of distinct macromolecules assemblages. Only a highly spatially resolved specialization of this tissue would be able to build a transition sequence, only a few tens of microns thick, that includes all the previously described steps.

The dotted pattern, enriched in organic molecules, that is found at the basis of crossed-lamellar layer is very intriguing: these spots may indeed play an important role in the initiation of the alternate orientation of 1st order lamellae-A still completely unexplained feature. Further dedicated investigations of organic/mineral interactions at these locations might prove very useful, as well as the characterization of their precise arrangement relatively to the 1st order lamellae disposition; the latter might however not be easily achieved, due to the relative complexity of crossed-lamellar pattern, and could ultimately require highly resolved 3D imaging techniques (such as $\mu$-tomography reconstructions or volume confocal microscopy).

\section{Experimental Section}

\subsection{Material}

Nerita undata (Linnaeus, 1758) (Gastropoda, Neritopsina) specimens were collected in the Tuamotu Archipelago, French Polynesia. The shells were cleaned using $\mathrm{NaClO}\left(10^{\circ}\right)$ solution for $15 \mathrm{~min}$, and then carefully rinsed with deionized water to remove remaining soft tissues. Radial sections were prepared on the peristome, close to the aperture of the shell, which forms a thickened lobe on the inner side of the outer lip, and specifically prepared according to the techniques subsequently used (detailed below).

\subsection{Methods}

\subsubsection{Polarized and Analyzed Light Microscopy}

Two sections were glued on a glass plate: one was cut and ground using HERMES water grinding papers (successively P1200/P2500 and P4000 grain sizes) until a standard $30 \mu \mathrm{m}$ thin thick section is reached, then polished using Buehler diamond polycrystalline suspensions ( $3 \mu \mathrm{m}$ and $1 \mu \mathrm{m})$. A second, much thinner section $(5-10 \mu \mathrm{m})$ was prepared using the same protocol. 


\subsubsection{Scanning Electron Microscopy}

Two radial sections were ground and polished using P1200, P2500 and P4000 HERMES water grinding papers, 3 and $1 \mu \mathrm{m}$ Buehler diamond polycrystalline suspensions, and aluminum oxide suspension (grain size $\sim 300 \mathrm{~nm}$ ). One section was prepared to stain organic compounds, thus enhancing organic/mineral contrast: it was first etched $20 \mathrm{~s}$ in a formic acid $(0.1 \% \mathrm{wt} . \%)$, glutaraldehyde (3\% v/v) solution, then successively stained (1) in $\mathrm{Pb}$ citrate solution for $30 \mathrm{~min}(0.4 \mathrm{~g}$ diluted in $100 \mathrm{~mL}$ of $\mathrm{H}_{2} \mathrm{O}$ and $10 \mathrm{~mL}$ of $\left.\mathrm{NaOH} 1 \mathrm{M}\right)$ and (2) in uranyl acetate solution for $20 \mathrm{~min}(6 \mathrm{~g}$ diluted in $90 \mathrm{~mL}$ of $\mathrm{H}_{2} \mathrm{O} /$ ethanol 50/50v). A second preparation was cut again, perpendicularly to the initial section, and the new transverse face was polished using the same protocol; both faces were then etched in a formic acid $(0.1 \%$ wt.\%), glutaraldehyde ( $3 \% \mathrm{v} / \mathrm{v})$ solution for $30 \mathrm{~s}$. Some freshly broken, unetched fragments were also prepared to illustrate microstructural organization of both layers.

Observations were carried out on (1) a Philips XL30 SEM using secondary (SE) and backscattered (BSE) electrons collectors and (2) a Carl Zeiss Ultra Plus Field Emission SEM using secondary electron (SE) collector. For (1), SEM was operated at $25 \mathrm{keV}$ and $10 \mathrm{~mm}$ working distance and sample was coated with $\mathrm{Au} / \mathrm{Pd}$. For (2), SEM was operated at $1 \mathrm{keV}$ and $4 \mathrm{~mm}$ working distance with an aperture of $10 \mu \mathrm{m}$ and sample was not coated.

\subsubsection{XANES Spectroscopy}

A section was embedded in epoxy resin and polished using P1200, P2500 and P4000 HERMES water grinding papers, 3 and $1 \mu \mathrm{m}$ Buehler diamond polycrystalline suspensions and carefully rinsed with deionized water, and then softly etched with acetic acid $(1 \% \mathrm{v} / \mathrm{v})$ for $5 \mathrm{~s}$ to remove any surface contamination.

Observations were carried out at the ID21 beamline of the European Synchrotron Radiation Facility (Grenoble, France). An energy range between 2 and $9 \mathrm{keV}$ is available, and the $\mathrm{X}$-ray beam energy was tuned for sulfur K-edge $(2,472 \mathrm{eV})$ using a fixed exit double-crystal $\mathrm{Si}(111)$ monochromator. The later provides an energy resolution of $\triangle \mathrm{E} / \mathrm{E}=10^{-4}$, necessary to resolve the micro XANES spectroscopy features, with a photon flux of $2.5 \times 10^{9}$ photon/s. The Scanning X-ray Microscope (SXM) [28] uses Fresnel zone plates as focusing optics to generate a sub-micron X-ray probe $(0.2 \times 0.7 \mu \mathrm{m})$, and was operated under vacuum to avoid absorption of the $\mathrm{S}$ emission lines by air. A high purity Ge energy dispersive detector (Princeton Gamma-Tech, New Jersey) mounted in the horizontal plane perpendicular to the beam was used to collect the X-ray fluorescence photons emitted from the sample. This geometry minimizes the contribution of elastic scattering.

Elements with absorption edges at lower energies than sulfur K-edge energy range are also excited, and emit X-ray fluorescence photons, allowing the determination of some elemental contents: the mapping of the distribution of strontium and phosphorus were therefore acquired simultaneously with the sulfur maps. These determinations are, however, only qualitative and by no means quantitative.

\subsubsection{FT-IR Spectroscopy}

One non-embedded section was polished and cleaned using the same protocol as for XANES experiment. Synchrotron-FT-IR measurements were performed at the ID21 beamline of the European 
Synchrotron Radiation Facility (Grenoble, France), on a Continuum IR microscope coupled to a Nexus Fourier transform IR bench (Thermo Nicolet). The focusing and collecting Schwarzschild objectives have a magnification of $32 \times$ (NA 0.65). The microscope is equipped with a computer-controlled stage for mapping purpose. Maps were acquired in reflection mode, using x/y steps of $10 \mu \mathrm{m}\left(10 \times 10 \mu \mathrm{m}^{2}\right.$ aperture size), and an accumulation of 256 spectral scans was performed at each step. Spectra were acquired within a 4,000 to $700 \mathrm{~cm}^{-1}$ range with a spectral resolution of $8 \mathrm{~cm}^{-1}$.

\subsubsection{Confocal Laser Microscopy}

For confocal microscopy, a section was polished using P1200, P2500 and P4000 HERMES water grinding papers, 3 and $1 \mu \mathrm{m}$ Buehler diamond polycrystalline suspensions, aluminum oxide suspension ( $\sim 300 \mathrm{~nm}$ size). The surface was kept unetched, but carefully rinsed with deionized water.

Observations were carried out on an Olympus FV-1000 inverted confocal microscope (housed at LIONS laboratory, CEA-Saclay, France). The sample surface was excited with a green He-Ne laser $(\lambda=543 \mathrm{~nm}$ ) and emission light was collected through a band pass BA $560 \mathrm{IF}$ filter. A $20 \times$ (Olympus UPLSAPO, NA $=0.75$ ) and a $40 \times$ (Olympus UPLFLN, NA $=0.75$ ) objectives were used, with a confocal aperture fixed at 80 and $130 \mu \mathrm{m}$, respectively. Scanning speed was set at $40 \mathrm{~ms} / \mathrm{line}$. All scans were focused $\sim 2 \mu \mathrm{m}$ under the sample surface to avoid any contribution of potential surface contamination or polishing remains.

\section{Acknowledgments}

We are most grateful to Corinne Chevallard from the "Laboratoire Interdisciplinaire sur l'Organisation Nanométrique et Supramoléculaire" (LIONS-CEA, Saclay, France) for all the help and expertise that she kindly provided for Laser Confocal Microscopy experiments.

The ESRF is thanked for granting beamtime under proposal No. EC442.

\section{References}

1. Bøggild, B. The shell structure of the molluscs. Der Kongelige Danske Videnskabernes Selskabs Skrifter, Naturvidenskabelig og Mathematisk Afdeling 1930, 9, 231-326.

2. Kobayashi, I. Internal shell microstructure of recent bivalvian Molluscs. Sci. Rep. Niigata Univ. 1971, E, 27-50.

3. Taylor, J.; Kennedy, W.; Hall, A. The shell structure and mineralogy of the Bivalvia.II. Lucinacea-Clavagellacea. Conclusions. Bull. Br. Mus. Nat. Hist. Zool. 1973, 22, 253-294.

4. Grégoire, C.; Duchateau, G.; Florkin, M. La trame protidique des nacres et des perles. Ann. Inst. Océanogr. 1955, 31, 1-36.

5. Mutvei, H. On the micro- and ultrastructure of the conchiolin in the nacreous layer of some recent and fossil molluscs. Stockholm Contrib. Geol. 1969, 20,1-17.

6. Krampitz, G.; Engels, J.; Cazaux, C. Biochemical studies on water-soluble proteins and related components of gastropod shells. In The Mechanisms of Mineralization in the Invertebrates and Plants; Watabe, N., Wilbur, K.M., Eds.; The Belle Baruch Library in Marine Science, University of South Carolina Press: Columbia, SC, USA, 1976; Volume 5, pp. 155-173. 
7. Cuif, J.P.; Dauphin, Y.; Denis, A.; Gaspard, D.; Keller, J.P. Continuité et périodicité du réseau organique intraprismatique dans le test de Pinna muricata Linné (Lamellibranches). C. R. Acad. Sci. Paris 1980, 290, 759-762.

8. Weiner, S.; Traub, W. Macromolecules in mollusc shells and their functions in biomineralization. Philos. Trans. R. Soc. Lond. B 1984, 304, 425-434.

9. Weiner, S. Biomineralization: A structural perspective. J. Struct. Biol. 2008, 163, 229-234.

10. Addadi, L.; Joester, D.; Nudelman, F.; Weiner, S. Mollusk shell formation: A source of new concepts for understanding biomineralization processes. Chem. Eur. J. 2006, 12, 980-987.

11. Nudelman, F.; Gotliv, B.; Addadi, L.; Weiner, S. Mollusk shell formation: Mapping the distribution of organic matrix components underlying a single aragonitic tablet in nacre. J. Struct. Biol. 2006, 153, 176-187.

12. Sethmann, I.; Putnis, A.; Grassmann, O.; Lobmann, P. Observation of nano-clustered calcite growth via a transient phase mediated by organic polyanions: A close match for biomineralization. Am. Mineral. 2005, 90, 1213-1217.

13. Wilmot, N.; Barber, D.; Taylor, J.; Graham, A. Electron microscopy of molluscan crossed-lamellar microstructure. Philos. Trans. R. Soc. Lond. B 1992, 337, 21-35.

14. Dauphin, Y.; Cuif, J.P.; Salomé, M.; Susini, J. Speciation and distribution of sulfur in a mollusk shell as revealed by in situ maps using X-ray absorption near-edge structure (XANES) spectroscopy at the S K-edge. Am. Mineral. 2005, 90, 1748-1758.

15. Pickering, I.; Prince, R.; Divers, T.; George, G. Sulfur K-edge X-ray absorption spectroscopy for determining the chemical speciation of sulfur in biological systems. FEBS Lett. 1998, 441, $11-14$.

16. Dauphin, Y.; Cuif, J.; Doucet, J.; Salomé, M.; Susini, J.; Williams, C. In situ mapping of growth lines in the calcitic prismatic layers of mollusc shells using X-ray absorption near-edge structure (XANES) spectroscopy at the sulphur K-edge. Mar. Biol. 2003, 142, 299-304.

17. De Stasio, G.; Schmitt, M.; Gellman, S. Spectromicroscopy at the organic-inorganic interface in biominerals. Am. J. Sci. 2005, 305, 673-686.

18. Cusack, M.; Dauphin, Y.; Cuif, J.P.; Salomé, M.; Freer, A.; Yin, H. Micro-XANES mapping of sulphur and its association with magnesium and phosphorus in the shell of the brachiopod, Terebratulina retusa. Chem. Geol. 2008, 253, 172-179.

19. Jones, G.; Jackson, B. Infrared Transmission Spectra of Carbonate Minerals; Springer: Berlin, Heidelberg, Germany, 1993.

20. Dauphin, Y. Infrared spectra and elemental composition in recent carbonate skeletons: Relationships between the 2 band wavenumber and $\mathrm{Sr}$ and $\mathrm{Mg}$ concentrations. Appl. Spectrosc. 1997, 51, 253-258.

21. Suzuki, M.; Dauphin, Y.; Addadi, L.; Weiner, S. Atomic order of aragonite crystals formed by mollusks. Cryst. Eng. Comm. 2011, 13, 6780-6786.

22. Dauphin, Y. Mineralizing matrices in the skeletal axes of two Corallium species (Alcyonacea). Comp. Biochem. Physiol. A Mol. Integr. Physiol. 2006, 145, 54-64.

23. Byler, D.; Susi, H. Examination of the secondary structure of proteins by deconvolved FTIR spectra. Biopolymers 1986, 25, 469-487. 
24. Krimm, S.; Bandekar, J. Vibrational spectroscopy and conformation of peptides, polypeptides, and proteins. Adv. Protein Chem. 1986, 38, 181-364.

25. Dauphin, Y.; Ball, A.D.; Cotte, M.; Cuif, J.P.; Meibom, A.; Salomé, M.; Susini, J.; Williams, C.T. Structure and composition of the nacre-prisms transition in the shell of Pinctada margaritifera (Mollusca, Bivalvia). Anal. Bioanal. Chem. 2008, 390, 1659-1669.

26. Nehrke, G.; Nouet, J. Confocal Raman microscope mapping as a tool to describe different mineral and organic phases at high spatial resolution within marine biogenic carbonates: Case study on Nerita undata (Gastropoda, Neritopsina). Biogeosciences 2011, 8, 3761-3769.

27. Nouet, J.; Baronnet, A.; Howard, L. Crystallization in organo-mineral micro-domains in the crossed-lamellar layer of Nerita undata (Gastropoda, Neritopsina). Micron 2011, 43, 456-462.

28. Susini, J.; Salomé, M.; Fayard, B.; Ortega, R.; Kaulich, B. The scanning X-ray microprobe at the ESRF X-ray microscopy beamline. Surf. Rev. Lett. 2002, 9, 203-212.

(C) 2012 by the authors; licensee MDPI, Basel, Switzerland. This article is an open access article distributed under the terms and conditions of the Creative Commons Attribution license (http://creativecommons.org/licenses/by/3.0/). 